\title{
Knockdown of miR-384-3p Protects Against Myocardial Ischemia-Reperfusion Injury in Rats Through Targeting HSP70
}

\author{
Chusheng Huang, Hailong Deng, Wen Zhao, Lei Xian \\ Department of Thoracic and Cardiovascular Surgery, The Second Affiliated Hospital of Guangxi Medical University, Nanning City, \\ Guangxi Zhuang Autonomous Region, 530007, China
}

\section{ABSTRACT}

Background: Myocardial infarction (MI) and heart failure remain critical states of heart disease with high mortality. Previous studies have indicated that miRNA has cardioprotective effects and can resist myocardial ischemia-reperfusion (I/R) injury. However, the role of mir-384-3p in MI has not been reported, and whether this miRNA can regulate the apoptosis of cardiomyocytes needs to be verified.

Methods: The effect of hypoxia-reperfusion (H/R) on cardiomyocyte activity was detected using MTT assay. MiR384-3p was knocked down or overexpressed in cardiomyocytes H/R models by pretreatment with miR-384-3p mimic or inhibitor to verify the function of miR-384-3p in H/R. Circulating levels of miR-384-3p was detected by quantitative realtime PCR, and protein expression was detected by western blotting. TUNEL staining and flow cytometry demonstrated a high degree of myocardium apoptosis after $\mathrm{H} / \mathrm{R}$ induction. Dual-Luciferase Reporter Assay detected dynamic expression of miR-384-3p and HSP70. The infarction size of $\mathrm{I} / \mathrm{R}$ rats was detected by 2,3,5-triphenyltetrazolium chloride (TTC) staining.

Results: MiR-384-3p was closely related to cardiomyocyte activity in H/R progression. Increased expression of mir384-3p can promote the production of cleaved caspase- 3 and cleaved PARP, thereby regulating cardiomyocyte apoptosis. HSP70 was a target of miR-384-3p and HSP70 silencing aggravated H/R-induced cardiomyocyte dysfunction. In an animal model, the expression level of HSP70 is regulated by miR-384-3p, and miR-384-3p inhibition remarkably reduced $\mathrm{I} / \mathrm{R}$-induced $\mathrm{MI}$ in rats.

Conclusion: In conclusion, the present report identified that HSP70 was a potential target of miR-384-3p, and miR-384-3p inhibition remarkably reduced I/R-induced MI in rats. Therefore, this study provides a novel therapeutic approach for the treatment of MI from bench to clinic.

Received November 2, 2020; accepted November 30, 2020

Correspondence: Lei Xian, Department of Thoracic and Cardiovascular Surgery, The Second Affiliated Hospital of Guangxi Medical University, No. 166 East University Road, Xixiangtang District, Nanning City, Guangxi Zhuang Autonomous Region, China, Telephone +86-07713240325 (e-mail: xianlei333@163.com)

\section{INTRODUCTION}

Myocardial infarction (MI) is myocardial ischemic necrosis caused by coronary artery occlusion. Although modern medical research has made important progress, $\mathrm{MI}$ and subsequent heart failure remain critical states of heart disease with high mortality [Heusch 2008; Mozaffarian 2016]. MI induces the apoptosis of cardiomyocytes and the activation and proliferation of cardiac fibroblasts, which lead to progressive cardiac fibrosis [Vainio 2019; Xia 2016]. Numerous patients with chronic myocardial ischemia related to atherosclerosis are not suitable for surgical treatment, thus, promoting neovascularization and cardiovascular function as a new therapeutic option [Kupatt 2010]. How to improve the microenvironment, promote angiogenesis, and further improve the perfusion of myocardial ischemic tissue to achieve the purpose of treatment has become a hot research topic.

The microRNA (miRNA) is a form of endogenous, noncoding RNA that plays a critical role in gene regulation and post-transcriptionally [Bhaskaran 2014; Rzeszutek 2020]. The functional miRNA molecules can inhibit protein expression and is associated with many diseases. Moreover, miRNAs are involved in multiple cellular, physiological, and pathological processes [Bartel 2018]. Previous studies have indicated that microRNA plays a major role in myocardial infarction [Peng 2014; Zhang 2015]. For instance, miR-24-3p has cardioprotective effects to resist myocardial ischemia-reperfusion (I/R) injury by suppressing RIPK1 [Tan 2018]. MiR-214 attenuates I/R-triggered myocardial dysfunction by suppressing PTEN and Bim1 expression [Wang 2016]. The increased expression of miR-15 can reduce the infarction size of I/R-induced MI [Hullinger 2012]. Some studies have found that miR-384-3p can promote apoptosis [Wang 2016], and the expression of miR-384-3p in I/R rats is significantly upregulated. However, the role of mir-384-3p in cardiomyocytes has not been reported, and whether this miRNA can regulate the apoptosis of cardiomyocytes needs to be verified.

The major function of heat shock proteins (HSPs) as molecular chaperones is to prevent protein misfolding and aggregation, which facilitates the proteins to separate in the native state and target lysosomal degradation under stress conditions [Becker 1994]. HSPs can improve the stability and function of some proteins by inhibiting their phosphorylation [Connarn 2014; Yu 2015]. The molecular chaperone 70 kilodalton heat shock proteins (HSP70) is one of the most widely studied proteins in the HSP family. HSP70 is a stress-inducible 
protein, which is overexpressed in diverse stress situations, such as oxidative stress or myocardial damage. HSP70 has been shown to sustainedly activate extracellular signal-regulated kinase 1/2 pathway and suppress the c-Jun N-terminal kinase 1signaling pathway, thereby suppressing cellular oxidative stress, attenuating the rise of calcium and reducing the ratio of cardiomyocytes apoptosis among I/R [Song 2019]. HSP70 can remarkably inhibit cardiomyocyte apoptosis after myocardial I/R injury [Peng 2010]. Recent studies have shown that HSP70 inhibits cardiomyocyte necrosis by inhibiting autophagy in myocardial I/R injury [Liu 2016]. Based on the given background, we explored the role of miR-384-3p in MI and verified its interaction with HSP70 to regulate the function of myocardial cells.

\section{METHODS}

Male Sprague-Dawley (SD) rats between 10-12 weeks of age (weighing $250 \pm 50 \mathrm{~g}$ ) were supplied by Shanghai Experimental Animal Center (Shanghai, China). Rats were housed under a temperature-controlled room with a 12-hour darklight cycle. All animal studies were approved by the Medical Ethics Committee of Second Affiliated Hospital of Guangxi Medical University and followed the U.S. National Institutes of Health Guidelines for the Care and Use of Laboratory Animals.

Animal model of acute myocardial infarction: To mimic acute myocardial infarction, the method of left coronary artery ligation was performed as previously described [Dong 2009]. In brief, SD rats were anesthetized with intraperitoneally $2 \%$ of the urethane. The rats were randomly separated in three experimental groups with 10 rats per group: sham operation control group (Sham), I/R + Ad-NC inh, and I/R + Ad-miR-134 inh. Animals were injected with adenovirus that expressed miR-384-3p or NC (negative control virus) and 7 days later, the same procedure described for I/R group was performed. In the Sham group, the animal's chest was opened except that their left anterior descending coronary arteries were not ligated. $\mathrm{I} / \mathrm{R}$ was achieved via treated with ischemia for 30 minutes and reperfusion for 24 hours of the left anterior descending coronary artery as described [Tang 2006].

Cell culture and ischemia injury model: The primary cultures of cardiomyocytes were obtained from neonatal rat cardiac ventricular and the cells were dispersed by digestion with collagenase type IV $(0.4 \mathrm{mg} / \mathrm{ml}), 0.1 \%$ trypsin, and $15 \mu \mathrm{g} / \mathrm{ml}$ DNase I. The cells were cultured in Dulbecco's modified Eagle's medium/F-12 supplemented with $10 \%$ fetal bovine serum and $1 \times 10^{5}$ cells per well were seeded into sixwell plates. The cardiomyocytes were transfected with miR384-3p mimic, miR-384-3p inhibitor, and negative control oligonucleotides and cultured in $3 \mathrm{~h}$ of reoxygenation after hypoxia for $4 \mathrm{~h}(\mathrm{H} / \mathrm{R})$.

Cell transfection: Cell transfections were executed by using Lipofectamine 2000 (Invitrogen, USA), according to the manufacturer's instructions. Primers used in this study were listed as following: the sense primer miR-384-3p mimic 5'-UCUUUGGUUAUCUAGCUGUAUGA-3', miR-384-3p inhibitor primer 5'-TCATACAGCTAGATAACCAAAGA-3', and negative control primer 5'-UUCUCCGAACGUGUCACGUTT-3'. The concentration of the miR-384-3p mimic, miR-384-3p inhibitor, and NC used for transfection was 100 $\mathrm{nM}$ in this study. The sequence of short hairpin RNA targeting Hsp70 was 5'-GCCCAAGGTGCAGGTGAACTA-3'.

Western blot: Cardiomyocytes were harvested and total protein from each group was separated by $10 \%$ SDS-PAGE and transferred onto polyvinylidene fluoride membranes. After incubated with blocking solution (5\% non-fat milk) for 1 hour, the membrane was incubated with 1:2000 diluted primary antibodies overnight at $4^{\circ} \mathrm{C}$. The antibodies against caspase 3, cleaved caspase 3 and PARP were purchased from Abcam (Cambridge, MA, USA). The antibodies against cleaved PARP, HSP70 and actin were obtained from Cell Signaling Technology (Beverly, MA, USA). After 1 hour of incubation with secondary antibody (1: 5000), chemiluminescence system was used for visualized and quantify the protein bands by Image J software.

RNA extraction, quantitative real time (RT)-PCR: Cardiomyocytes were harvested and total RNAs were isolated by TRIzol reagent (Invitrogen). The total RNA was reverse transcribed by using universal cDNA synthesis and the q-RTPCR was performed by using SYBR Green Master Mix kits. The expression of miR-384-3p was normalized to U6. Fold change is expressed over sham control tissue. RNA expression data calculated by the comparative $\mathrm{Ct}$ method $\left(2^{-\Delta \Delta \mathrm{Ct}}\right)$ as described by the previous study [Ke 2015]. The primers used in present study were listed as following:

miR-384-3p:

Forward $\left(5^{\prime}-3^{\prime}\right)$ : TTTTAGAAGCGTTCGGCCAAA; Reverse (5'-3'): ACCAGAGTTGCCATTGATAGTGA; U6:

Forward (5'-3'): TCCTCCACGACAACCAAAACC;

Reverse $\left(5^{\prime}-3^{\prime}\right)$ : TCTTTTCCCAAAATCCCAGACTC.

2,3,5-Triphenyltetrazolium chloride staining: After treatment, the rats were anesthetized, and the heart tissues were frozen and cut into 5 transverse slices from apex to base of equal thickness $(2 \mathrm{~mm})$. The sections were counterstained with 2,3,5-triphenyltetrazolium chloride (TTC) solution for 15 minutes and fixed with 4\% neutral paraformaldehyde. Viable myocardium was red, whereas the infarct area was white. The area of myocardial infarction was analyzed with Image-Pro 6.0.

Flow cytometry: Cardiomyocytes were detached with trypsin and $1 \times 10^{6} / \mathrm{ml}$ cells were resuspended in binding buffer. Then cells were incubated with $5 \mu$ Annexin V-FITC and $5 \mu \mathrm{l}$ propidium iodide from Alexa Fluor ${ }^{\circledR} 488$ Annexin V/Dead Cell Apoptosis Kit (Thermo, USA), following the manufacturer's instructions. The portion of apoptotic cells in the experimental group was compared with that of the control group in each experiment.

Dual luciferase reporter gene assay: To investigate whether HSP70 is a target of miR-384-3p, the binding sites of miR384-3p and HSP70 were forecasted by DianaTools. pGL3 luciferase reporter gene vector (Promega, Madison, WI, USA) loaded with wild type (WT)-HSP70 or Mutant (mut)-HSP70 were co-transfected with miR-384-3p mimic, miR-384-3p 
inhibitor or negative control (NC) into cardiomyocytes. The luciferase activity was assayed with the dual luciferase reporter assay system (Promega, WI, USA), according to the manufacturer's instructions.

MTT assay and LDH analysis: 3-(4,5-Dimethylthiazol2-yl)-2,5-diphenyltetrazolium bromide (MTT) assay (Abcam, Cambridge, UK) were used to detect cardiomyocytes proliferation in the different groups, according to the manufacturer's instructions. Lactate dehydrogenase (LDH) release was detected by using the supernatants of cardiomyocytes by a commercial LDH cytotoxicity assay kit (Thermo, USA). The cell vitality and the absorbance were measured using a microplate reader (Thermo, USA) at $490 \mathrm{~nm}$.

TUNEL staining: Cell apoptosis was measured by TUNEL Assay Kit (Abcam, Cambridge, UK), following manufacturer's instructions. Cardiomyocytes were cultured in 6-well plates, and the measurement was managed when the cells were cultured to $80 \%$ confluence. The nuclear morphology was stained with 4,6-diamidino-2-phenylindole (DAPI). The sample's image was acquired by a fluorescence microscope. Nuclei labeled with DAPI and TUNEL were considered positive. Apoptosis rate $(\%)=$ the number of TUNEL positive myocytes/total number of myocytes $\times 100 \%$

Statistical analysis: All data were analyzed using Statistical Product and Service Solutions (SPSS) 19.0 (SPSS Inc., Chicago, IL, USA) software package. Data were expressed as mean \pm SD. The differences among groups were performed with one-way ANOVA followed by Tukey's post hoc test. $P$-value $<0.05$ was defined as statistically significant.

\section{RESULTS}

MiR-384-3p is upregulated in myocardial $H / R$ injury: MTT assay was performed to evaluate the effect of H/R on cardiomyocyte activity, and the results showed that the activity of cardiomyocytes was remarkably inhibited after H/R stimulation. The viability of rat cardiomyocytes was substantially decreased after $H / R$ treatment (Figure $1 \mathrm{~A}$ ), whereas the expression level of mir-384-3p was considerably increased. (Figure 1) qRT-PCR was introduced to analyze the levels of miR-384-3p. Compared with normal cardiomyocytes, miR384-3p was upregulated in H/R-injured cardiomyocytes (Figure 1B). These results indicated that miR-384-3p was closely related to cardiomyocyte activity in H/R progression.

Knockdown of miR-384-3p attenuates $H / R$-induced cellular apoptosis: The function of miR-384-3p in H/R in $\mathrm{H} / \mathrm{R}$ models was examined by pretreatment with miR-384-3p mimic or inhibitor to modify the expression level of miR384-3p. The results indicated that mir-384-3p inhibitor could reverse the decrease in cardiomyocytes activity induced by H/R. Meanwhile, miR-384-3p mimic could further aggravate the decrease in cardiomyocyte activity induced by H/R (Figure 2A). (Figure 2) TUNEL staining and Annexin V-FITC apoptosis flow cytometry results demonstrated a high degree of myocardium apoptosis after $\mathrm{H} / \mathrm{R}$ induction; however, miR384-3p inhibitor substantially alleviated H/R-induced apoptosis. Interestingly, miR-384-3p mimic can further aggravate
A

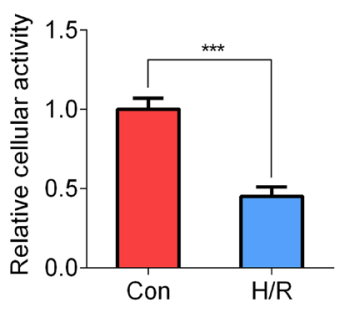

Figure 1. Upregulation of miR-384-3p is involved in H/R-induced cardiomyocyte injury. (A) MTT assay was used to detect the cell viability of each group. (B) miR-384-3p expression in cardiomyocytes was assessed by $\mathrm{qRT}-\mathrm{PCR}$. *** $P<0.001$ vs. control. Data are expressed as mean $\pm \mathrm{SD} ; N=3$.

H/R-induced myocardium apoptosis (Figure 2B) (Figure 2C). In addition, the expression levels of cleaved caspase- 3 and cleaved PARP were remarkably increased after H/R induction, but the apoptotic ratios considerably decreased after the miR-384-3p inhibitor treatment. Conversely, miR-384-3p mimic increased the apoptotic ratio (Figure 2D). These results suggested that the increased expression of mir-384-3p can promote the production of cleaved caspase- 3 and cleaved PARP, thereby regulating cardiomyocyte apoptosis.

MiR-384-3p functions as an HSP70 regulator: HSP70 is a cardioprotective substance that can inhibit myocardial cell apoptosis after $\mathrm{H} / \mathrm{R}$ injury. To predict the target genes of miR384-3p, we used TargetScan (http://www.targetscan.org), and the results indicated that the HSP70 gene constituted a miR384-3p-binding domain on its 3' UTR (Figure 3A). (Figure 3) We performed a miRNA target validation experiment by cotransfecting cells with either a HSP70-wt or HSP70-mut vector and either miR-384-3p mimic, miR-384-3p inhibitor, or their negative control (NC). Dual-luciferase reporter assays showed that compared with NC, the luciferase activity of HSP70-wt was remarkably decreased in cardiomyocytes transfected with miR-384-3p mimic and markedly increased in cardiomyocytes transfected with miR-384-3p inhibitor. No substantial difference in luciferase activity was found in the HSP70-mut group (Figure 3B). Next, the relationship between miR-384-3p and HSP70 were determined by Western blot (Figure 3C). We found that $H / R$ induction could increase the expression of HSP70. H/R induction with miR-384-3p mimic treatment remarkably inhibited the protein expression of HSP70, while miR-384-3p silencing treatment reversed its suppression effect and promoted the expression of HSP70. The results showed that HSP70 is a target axis of miR-384-3p, and miR-384-3p can inhibit the expression of HSP70.

HSP70 knockdown reverses the protective effect of miR384-3p knockdown on H/R-induced cardiomyocyte injury. HSP70 was silenced in cardiomyocytes following H/R injury to investigate the role of HSP70 in the protective effect of miR-384-3p knockdown on H/R. The results showed that HSP70 silencing could further reduce the H/R-induced decrease in cell viability (Figure 4A). (Figure 4) However, compared with miR-384-3p knockdown alone, cell viability 
A

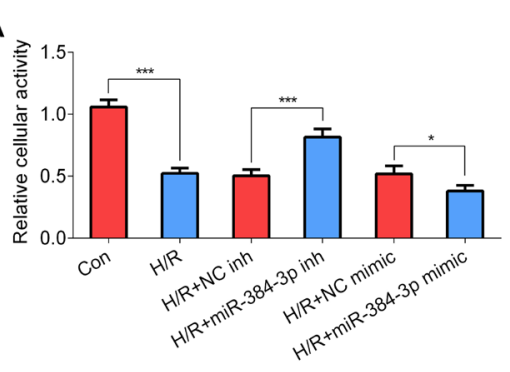

B

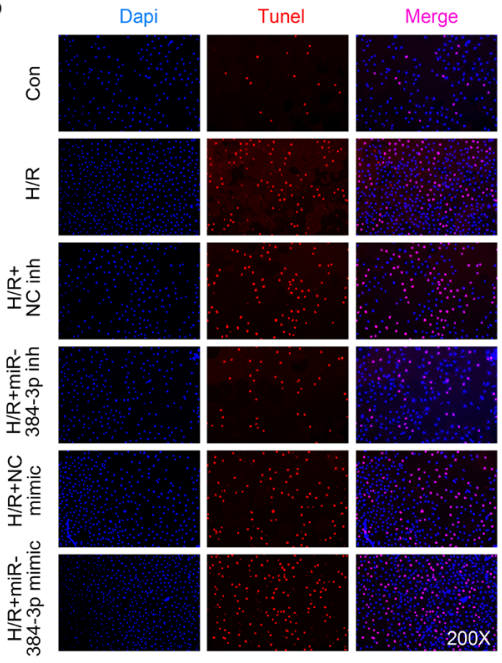

C
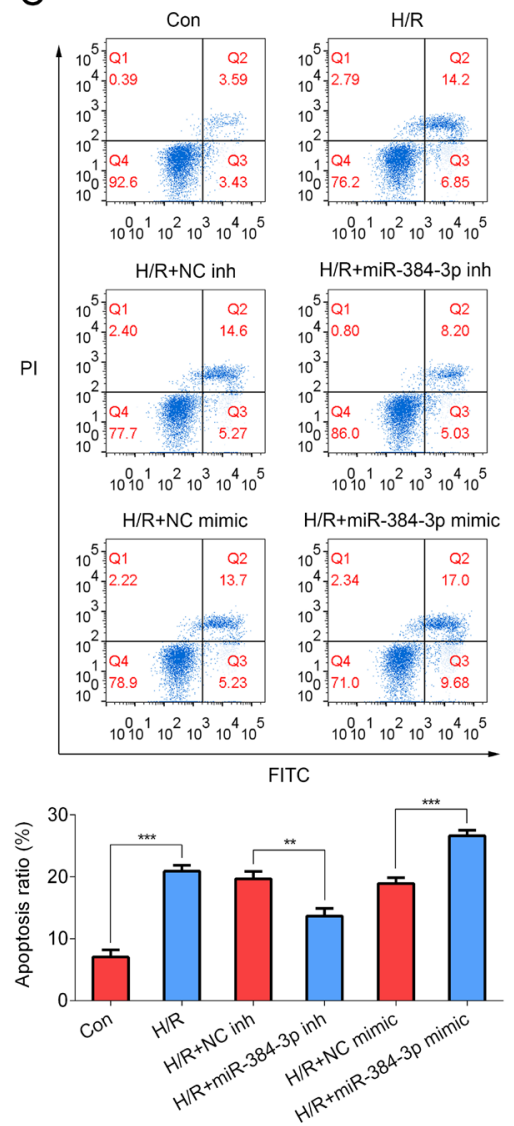

D
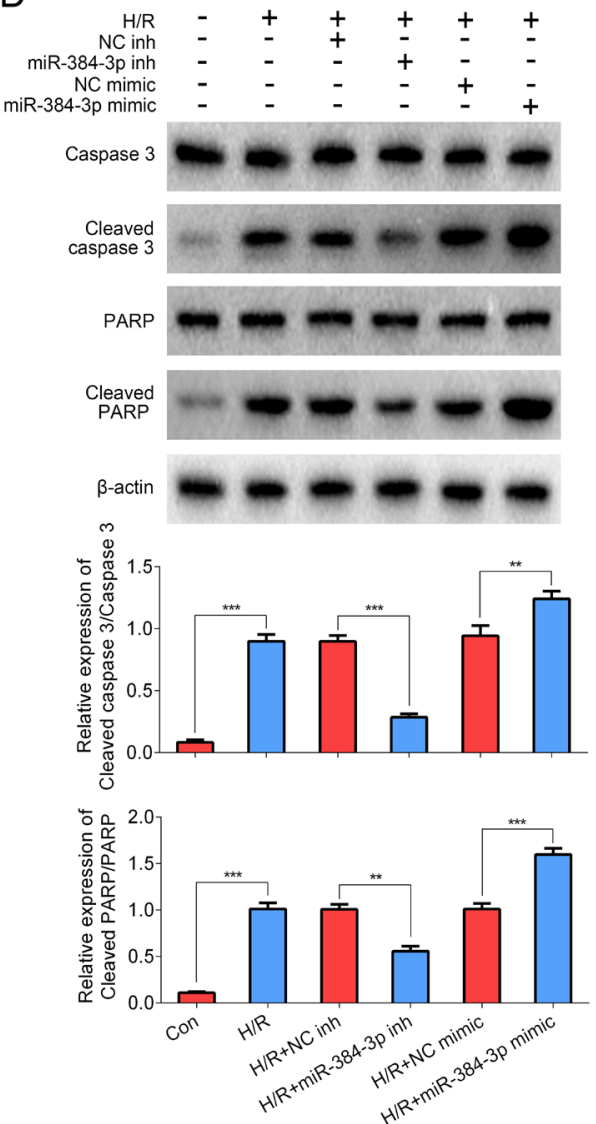

Figure 2. Knockdown of miR-384-3p reduces $\mathrm{H} / \mathrm{R}$-induced cardiomyocytes apoptosis. (A) MTT assay was used to detect the cell viability of each group. Cardiomyocytes were allocated to 6 groups: control, $\mathrm{H} / \mathrm{R}, \mathrm{H} / \mathrm{R}+\mathrm{miR}-384-3 \mathrm{p}$ mimic, $\mathrm{H} / \mathrm{R}+\mathrm{NC}$ mimic, $\mathrm{H} / \mathrm{R}+\mathrm{miR}-384-3 \mathrm{p}$ inhibitor and $\mathrm{H} / \mathrm{R}+\mathrm{NC}$ inhibitor. (B) Representative immunofluorescence photomicrographs of and TUNEL (apoptosis marker, red) positive cells in each experimental group. All cell nuclei were counterstained with DAPI (blue). (C) Flow cytometry measured apoptosis of cardiomyocytes. (D) Western blotting analysis for the apoptosis-related protein expression (cleaved PARP, PARP, cleaved caspase-3 and caspase-3). Data are expressed as mean \pm SD; $N=3$. *P<0.05; $* * P<0.01$; $* * *<0.001$.

was remarkably decreased when miR-384-3p inhibitor and HSP70 silencing were transfected at the same time (Figure 4A). The apoptosis experiments also showed that HSP70 silencing could substantially reverse the protective effect of miR-384-3p knockdown on the ratio of cardiomyocyte apoptosis (Figure 4B) (Figure 4C). Similarly, compared with miR384-3p inhibition alone, knockdown HSP70 enhanced the expression of cleaved caspase- 3 and cleaved PARP, but this effect was remarkably reversed when miR-384-3p and HSP70 were both inhibited (Figure 4D). These results indicated that HSP70 silencing can aggravate H/R-induced cardiomyocyte dysfunction, whereas inhibiting miR-384-3p could reverse the effect of HSP70 silencing on cardiomyocytes.

MiR-384-3p knockdown suppresses I/R-induced MI in rats: Simultaneously, miR-384-3p was silenced in $I / R$ models by pretreatment with miR-384-3p inhibitor adenovirus to explore the effect of miR-384-3p on myocardial I/R injury. As shown in Figure 5A, the infarction size of $\mathrm{I} / \mathrm{R}$ rats was remarkably increased, and the infarction size tended to decrease after the inhibition of miR-384-3p. (Figure 5)
LDH activity and miR-384-3p expression level in I/R rats were substantially increased; however, after the inhibition of miR-384-3p, the activity of LDH activity was remarkably decreased and miR-384-3p was almost not expressed in the myocardial tissues of rats (Figure 5B) (Figure 5C). The results of Western blot showed that the expression levels of HSP70 protein, cleaved caspase-3, and cleaved PARP in I/R rats were remarkably increased. After inhibiting miR-384-3p, the expression level of HSP70 protein was further increased whereas the expression levels of cleaved caspase-3 and cleaved PARP were decreased (Figure 5D). Taken together, these results suggest that HSP70 expression level was regulated by miR-384-3p, and miR-384-3p inhibition remarkably reduced $\mathrm{I} / \mathrm{R}$-induced $\mathrm{MI}$ in rats.

\section{DISCUSSION}

In this study, our results for the first time showed that the HSP70 expression level is regulated by miR-384-3p, and 
A

HSP70-WT 5'...ACAUGAGACAAAGUUUAGGAAAA...3'

rno-miR-384-3p 3' UAACACUUGUUAAAGAUCCUUA 5'

HSP70-MUT 5'...ACAUGAGACAAAGUUAUCCUUAA...3'

B

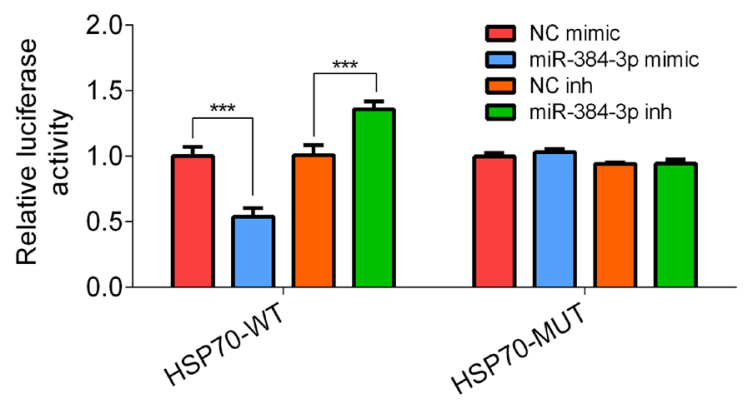

C
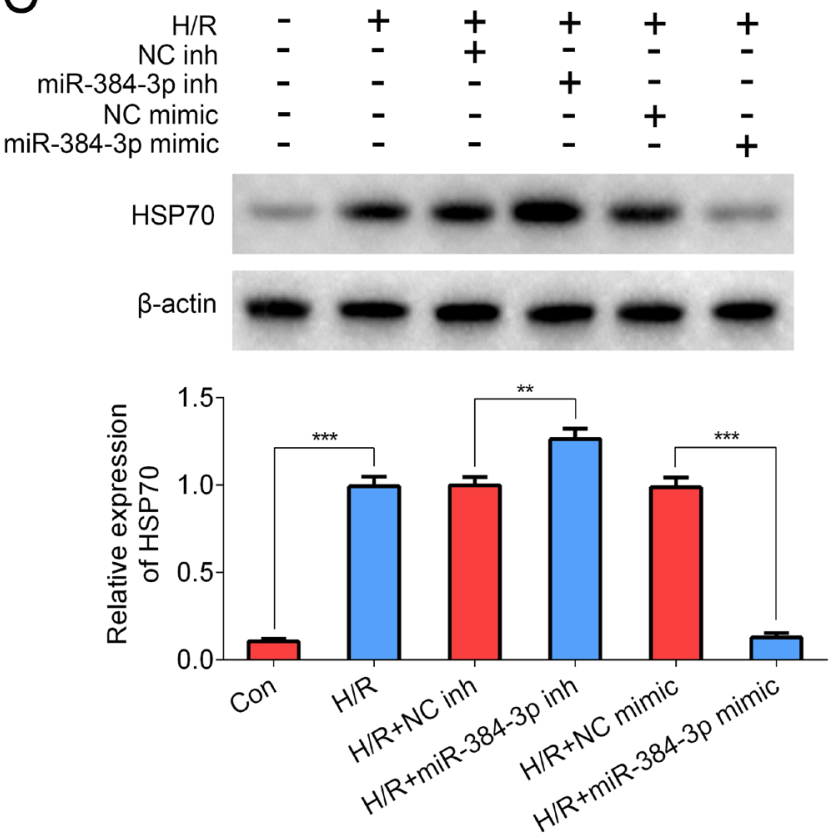

Figure 3. miR-384-3p directly targets HSP70. (A) Wild-type (HSP70-wt) and mutant (HSP70-mut) miR-384-3p binding sites in the 3'UTR of HSP70. (B) Dual-luciferase activity assay was performed at 36h after cardiomyocytes were co-transfected with HSP70-wt or HSP70-mut vector and miR-384-3p mimic, miR-384-3p inhibitor or their negative control (NC). (C) HSP70 protein was examined by Western blot analysis. Data are expressed as mean \pm SD; $N=3$. $* * P<0.01 ; * * * P<0.001$.

miR-384-3p inhibition remarkably reduced I/R-induced MI.

Reperfusion of ischemic tissues, termed as I/R injury, induces cardiomyocyte death, leading to impaired cardiac function. I/R injury is caused by depriving of blood supply to the tissue for a period of time and by cellular damage occurring as a consequence of reperfusion. Numerous studies have investigated the essential molecular mechanisms of myocardial I/R-injury to find effective therapeutic approaches to reduce $\mathrm{I} / \mathrm{R}$ injury-mediated cardiomyocyte apoptosis [Maneechote 2018; Zhu 2015]. Recently, research has reported the use of miRNAs to effectively diagnose and treat cardiovascular diseases, such as MI, atherosclerosis, and hypertension [de Lucia 2017; Samanta 2016]. Some cardiac miRNAs, such as miR-1, miR-208a, miR-499, miR-499-5p, and miR-133, were found to be invariably upregulated in the plasma of patients with acute MI within hours after the onset of infarction [Pinchi 2019; Xiao 2014]. Previous studies have demonstrated that miRNAs (miR-409-3p, miR-384-3p, and miR-101a) are involved in myocardial disease [Kim 2019]. Consistent with this finding, a preliminary study indicated that the expression level of mir-384-3p is remarkably increased in I/R-induced cardiomyocytes [Kim 2019]. In this study, the results suggest that overexpression of miR-384-3p may be a missing link in the poor prognosis of acute MI. The apoptosis of cardiomyocytes is closely related to the severity of acute MI. Thus, we explored the effects of modified expression of miR-384-3p in a I/R model on regulation of cardiomyocyte apoptosis. The inhibition of miR-384-3p in cardiomyocytes attenuated the cellular apoptosis induced by
I/R injury. Although miR-384-3p is a biomarker with clinical importance in acute MI diagnosis [Borosch 2017], the mechanism that regulates miRNA function has not been completely explained. Bioinformatic algorithms were used to search for potential target genes of miR-384-3p to identify the mechanisms involved in the miR-384-3p-mediated suppression of I/R injury. TargetScan results showed that the HSP70 gene constitutes a miR-384-3p-binding domain on its 3' UTR. HSP70 exerts anti-apoptotic function in downstream signaling pathways, which activate caspase-3-like proteases and prevent $\mathrm{Bcl} 2$-associated $\mathrm{X}$ translocation [Bari 2003; Ribeil 2007]. Furthermore, overexpression of HSP70 also prevents proteins misfolding and aggregating. In this study, HSP70 silencing aggravated I/R-induced cardiomyocyte dysfunction, whereas inhibiting miR-384-3p reversed HSP70 silencing-induced effects on cardiomyocytes. These results are consistent with previous reports that the overexpression of HSP70 could attenuate heart and brain I/R injury. Furthermore, HSP70 expression level is regulated by $\mathrm{miR}-384-3 \mathrm{p}$, and miR-384-3p inhibition remarkably reduced I/R-induced $M I$ in rats.

In conclusion, the present report demonstrated that the knockdown of miR-384-3p protected cardiomyocytes from I/R-induced cellular dysfunction and apoptosis. Moreover, these results identified that HSP70 is a potential target of miR-384-3p. HSP70 expression level is regulated by miR384-3p, and miR-384-3p inhibition remarkably reduced I/Rinduced $\mathrm{MI}$ in rats. Therefore, this study provides a potential therapeutic target for the treatment of MI. 
A

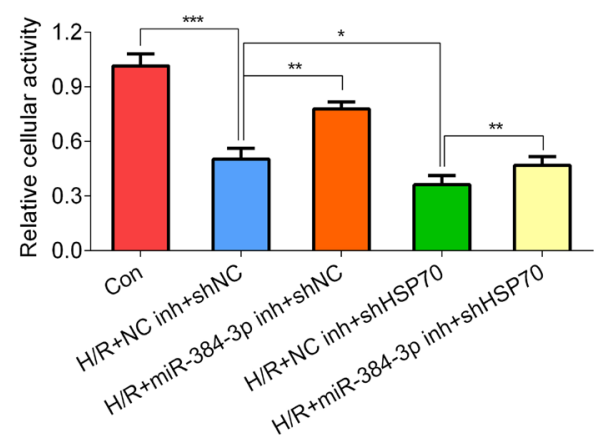

B

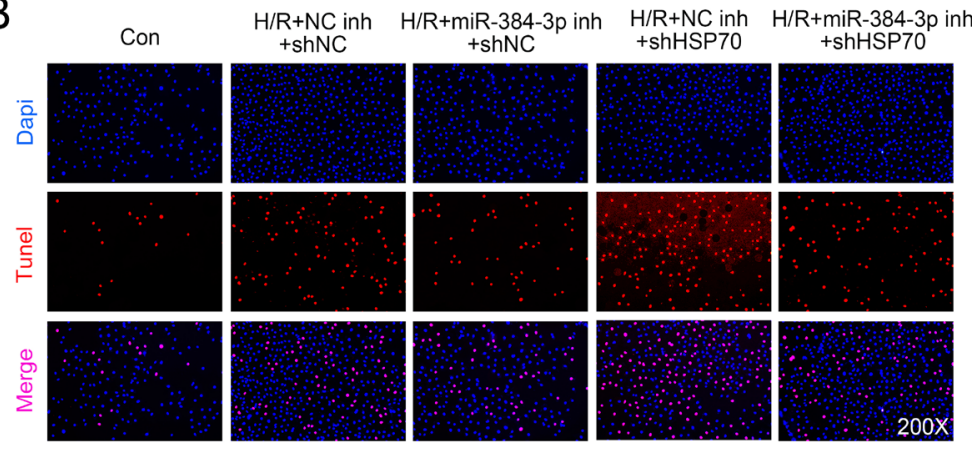

C

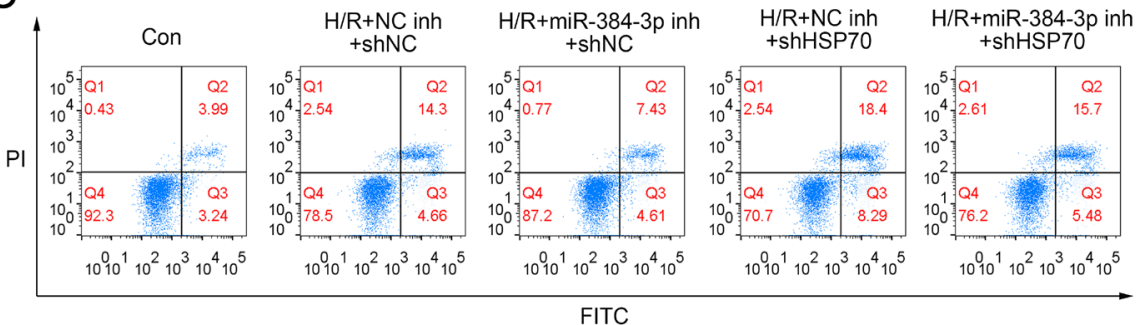

FITC

\section{D}

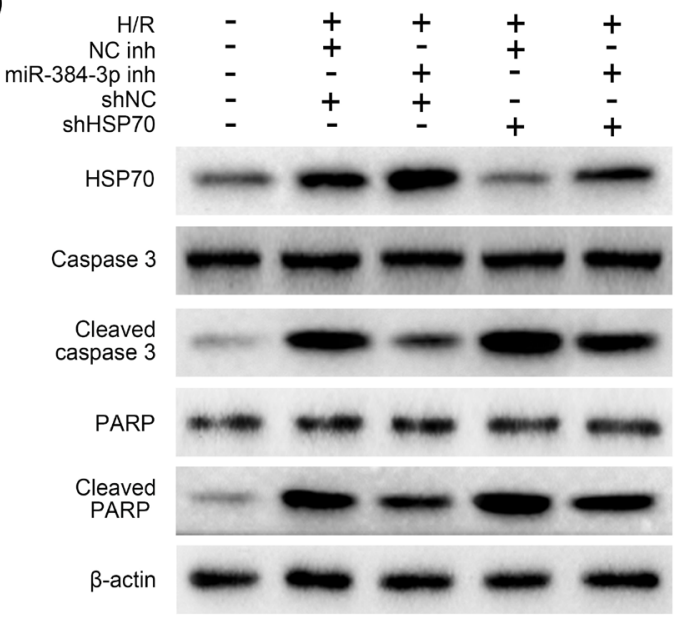

$\square$ Con

口 $\mathrm{H} / \mathrm{R}+\mathrm{NC}$ inh+shNC

H/R+miR-384-3p inh+shNC

$\square \mathrm{H} / \mathrm{R}+\mathrm{NC}$ inh+shHSP70

口 H/R+miR-384-3p inh+shHSP70
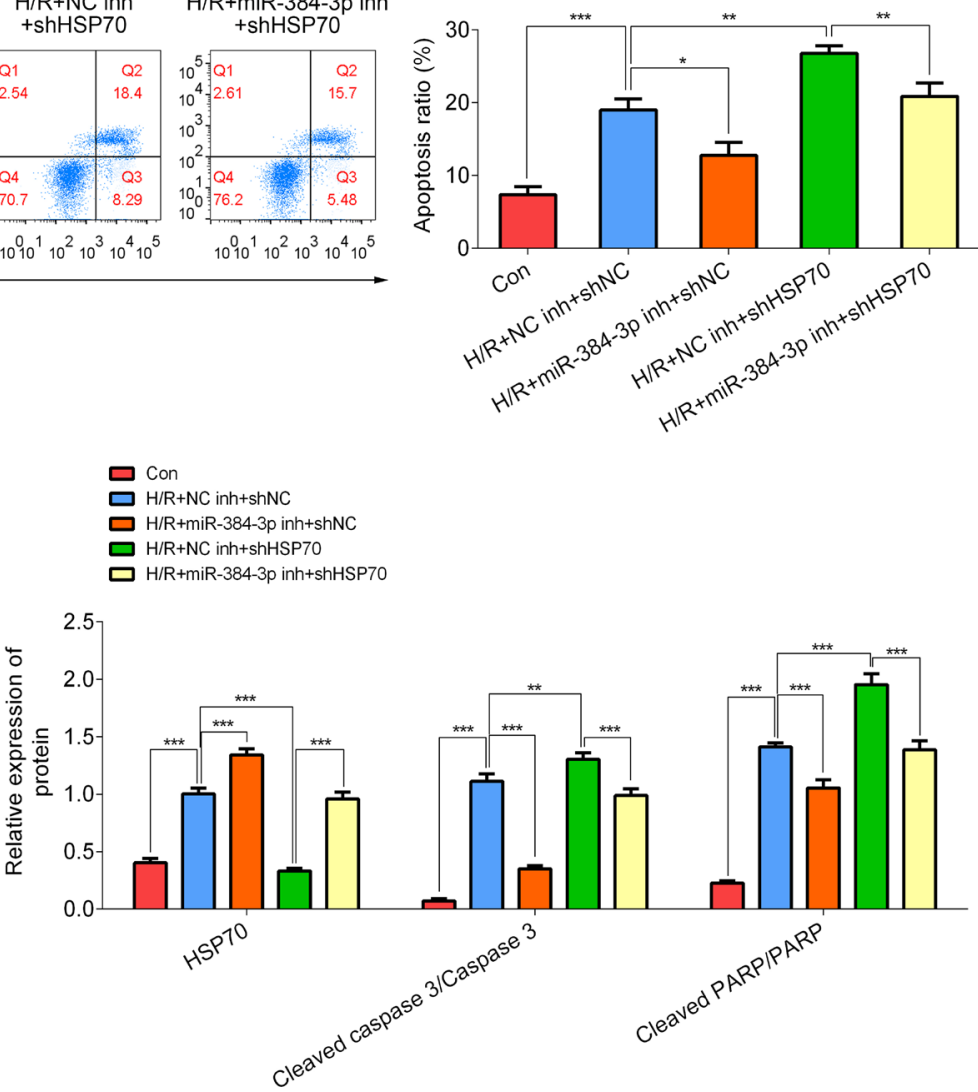

Figure 4. miR-384-3p regulated cardiomyocyte injury induced by $H / R$ through HSP70. (A) MTT assay was used to detect the cell viability of each group. Cardiomyocytes were allocated to 5 groups: control, $\mathrm{H} / \mathrm{R}+\mathrm{NCinh}+\mathrm{shNC}, \mathrm{H} / \mathrm{R}+\mathrm{miR}-384-3 p$ inh + shNC, $\mathrm{H} / \mathrm{R}+\mathrm{NCinh}+\operatorname{shHSP70}$ and $\mathrm{H} / \mathrm{R}+\mathrm{miR}-384-3 p$ inh + shHSP70. (B) Representative immunofluorescence photomicrographs of and TUNEL (apoptosis marker, red) positive cells in each experimental group. All cell nuclei were counterstained with DAPI (blue). (C) Flow cytometry measured apoptosis of cardiomyocytes. (D) Western blotting analysis for HSP70 and the apoptosis-related protein expression (cleaved PARP, PARP, cleaved caspase-3 and caspase-3). Data are expressed as mean $\pm S D ; N=3$. * $P<0.05$; $* * P<0.01 ; * * * P<0.001$. 
A

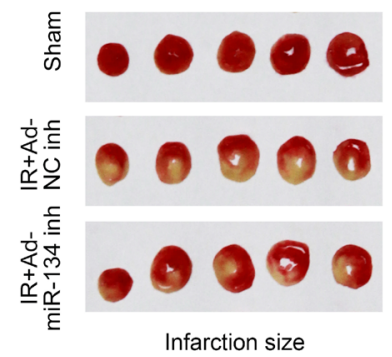

B

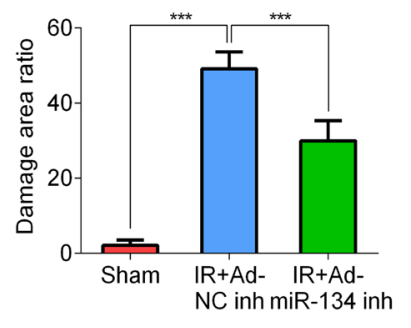

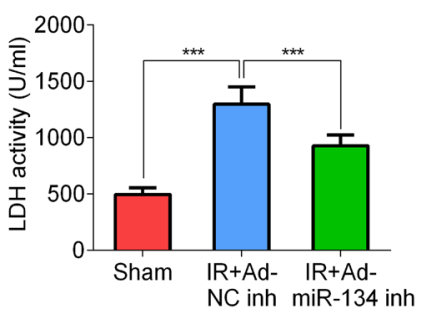

C

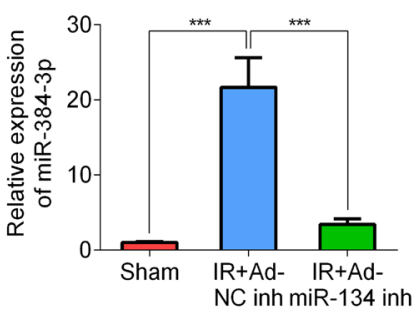

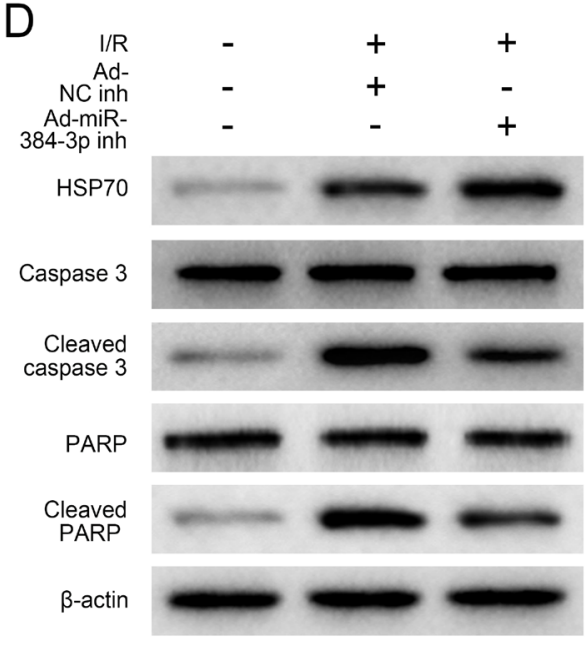

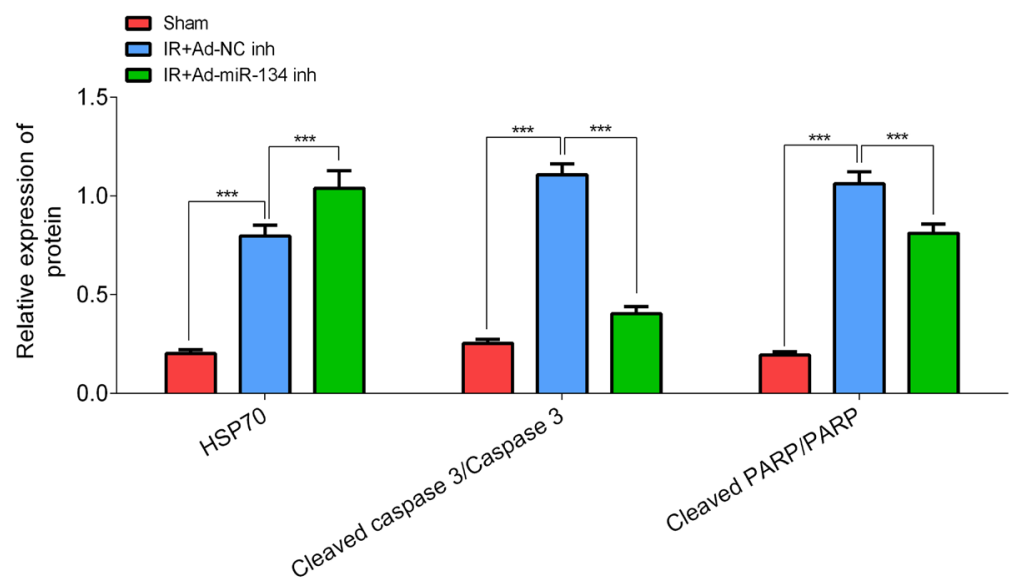

Figure 5. Knockdown of miR-384-3p suppressed ischemia/reperfusion induced myocardial infarct in rats. (A) Representative cross-sectional images of I/R (ischemia 30min, reperfusion 24h) heart with TTC. $N=10$. (B) Detection of serum lactate dehydrogenase (LDH) level. (C) The expression level of miR-384$3 p$ in vivo was measured by qRT-PCR. (D) The expression of HSP70, caspase 3, cleaved-caspase 3, PARP and cleaved-PARP were measured by western blot. Data are expressed as mean $\pm S D ; N=10 . * * P<0.01 ; * * P<0.001$.

\section{ACKNOWLEDGEMENT}

This work was supported by the Science Foundation of The Health Commission of Guangxi Zhuang Autonomous Region (Grant No. Z20181002). Ethical approval was obtained from the Medical Ethics Committee of Second Affiliated Hospital of Guangxi Medical University.

\section{REFERENCES}

Bari K, Petrik J, Rumora L. 2003. Biochemistry of apoptotic cell death. Acta pharm 53:151-164.

Bartel D. 2018. Metazoan MicroRNAs. Cell 173:20-51.

Becker J, Craig E. 1994. Heat-shock proteins as molecular chaperones. European journal of biochemistry 219:11-23.

Bhaskaran M, Mohan M. 2014. MicroRNAs: history, biogenesis, and their evolving role in animal development and disease. Veterinary pathology 51:759-774.
Borosch S, Dahmen E, Beckers C, Stoppe C, Buhl EM, Denecke B, et al. 2017. Characterization of extracellular vesicles derived from cardiac cells in an in vitro model of preconditioning. Journal of extracellular vesicles 6:1390391.

Connarn J, Assimon V, Reed R, Tse E, Southworth D, Zuiderweg E, et al. 2014. The molecular chaperone Hsp70 activates protein phosphatase 5 (PP5) by binding the tetratricopeptide repeat (TPR) domain. The Journal of biological chemistry 289:2908-2917.

de Lucia C, Komici K, Borghetti G, Femminella GD, Bencivenga L, Cannavo A, et al. 2017. microRNA in cardiovascular aging and agerelated cardiovascular diseases. Frontiers in medicine 4:74.

Dong S, Cheng Y, Yang J, Li J, Liu X, Wang X, et al. 2009. MicroRNA expression signature and the role of microRNA-21 in the early phase of acute myocardial infarction. The Journal of biological chemistry 284:29514-29525.

Heusch G, Boengler K, Schulz R. 2008. Cardioprotection: nitric oxide, protein kinases, and mitochondria. Circulation 118:1915-1919.

Hullinger T, Montgomery R, Seto A, Dickinson B, Semus H, Lynch J, et al. 2012. Inhibition of miR-15 protects against cardiac ischemic injury. Circulation research 110:71-81. 
Ke J, Yao Y-1, Zheng J, Wang P, Liu Y-h, Ma J, et al. 2015. Knockdown of long non-coding RNA HOTAIR inhibits malignant biological behaviors of human glioma cells via modulation of miR-326. Oncotarget 6:21934.

Kim EN, Kim CJ, Kim SR, Song J-A, Choe H, Kim K-B, et al. 2019. High serum CRP influences myocardial miRNA profiles in ischemiareperfusion injury of rat heart. PLoS One 14:e0216610.

Kupatt C, Hinkel R, Pfosser A, El-Aouni C, Wuchrer A, Fritz A, et al. 2010. Cotransfection of vascular endothelial growth factor-A and platelet-derived growth factor-B via recombinant adeno-associated virus resolves chronic ischemic malperfusion role of vessel maturation. Journal of the American College of Cardiology 56:414-422.

Liu X, Zhang C, Zhang C, Li J, Guo W, Yan D, et al. 2016. Heat shock protein 70 inhibits cardiomyocyte necroptosis through repressing autophagy in myocardial ischemia/reperfusion injury. In vitro cellular \& developmental biology Animal 52:690-698.

Maneechote C, Palee S, Kerdphoo S, Jaiwongkam T, Chattipakorn SC, Chattipakorn N. 2018. Differential temporal inhibition of mitochondrial fission by Mdivi-1 exerts effective cardioprotection in cardiac ischemia/ reperfusion injury. Clinical Science 132:1669-1683.

Mozaffarian D, Benjamin E, Go A, Arnett D, Blaha M, Cushman M, et al. 2016. Executive Summary: Heart Disease and Stroke Statistics--2016 Update: A Report From the American Heart Association. Circulation $133: 447-454$.

Peng L, Chun-guang Q, Bei-fang L, Xue-zhi D, Zi-hao W, Yun-fu L, et al. 2014. Clinical impact of circulating miR-133, miR-1291 and miR$663 \mathrm{~b}$ in plasma of patients with acute myocardial infarction. Diagnostic pathology 9:89.

Peng W, Zhang Y, Zheng M, Cheng H, Zhu W, Cao C, et al. 2010. Cardioprotection by CaMKII-deltaB is mediated by phosphorylation of heat shock factor 1 and subsequent expression of inducible heat shock protein 70. Circulation research 106:102-110.

Pinchi E, Frati P, Aromatario M, Cipolloni L, Fabbri M, La Russa R, et al. 2019. miR-1, miR-499 and miR-208 are sensitive markers to diagnose sudden death due to early acute myocardial infarction. Journal of cellular and molecular medicine 23:6005-6016.

Ribeil J-A, Zermati Y, Vandekerckhove J, Cathelin S, Kersual J, Dussiot M, et al. 2007. Hsp70 regulates erythropoiesis by preventing caspase3-mediated cleavage of GATA-1. Nature 445:102-105.

Rzeszutek I, Singh A. 2020. Small RNAs, Big Diseases. International journal of molecular sciences 21:

Samanta S, Balasubramanian S, Rajasingh S, Patel U, Dhanasekaran A, Dawn B, et al. 2016. MicroRNA: a new therapeutic strategy for cardiovascular diseases. Trends in cardiovascular medicine 26:407-419.

Song Y, Zhong C, Wang X. 2019. Heat shock protein 70: A promising therapeutic target for myocardial ischemia-reperfusion injury. Journal of cellular physiology 234:1190-1207.

Tan H, Qi J, Fan B, Zhang J, Su F, Wang H. 2018. MicroRNA-24-3p Attenuates Myocardial Ischemia/Reperfusion Injury by Suppressing RIPK1 Expression in Mice. Cellular physiology and biochemistry : international journal of experimental cellular physiology, biochemistry, and pharmacology 51:46-62.

Tang X, Sato H, Tiwari S, Dawn B, Bi Q, Li Q, et al. 2006. Cardioprotection by postconditioning in conscious rats is limited to coronary occlusions < $45 \mathrm{~min}$. American journal of physiology Heart and circulatory physiology 291:H2308-2317.

Vainio L, Szabó Z, Lin R, Ulvila J, Yrjölä R, Alakoski T, et al. 2019. Connective Tissue Growth Factor Inhibition Enhances Cardiac Repair and Limits Fibrosis After Myocardial Infarction. JACC Basic to translational science 4:83-94.

Wang X, Ha T, Hu Y, Lu C, Liu L, Zhang X, et al. 2016. MicroRNA-214 protects against hypoxia/reoxygenation induced cell damage and myocardial ischemia/reperfusion injury via suppression of PTEN and Bim1 expression. Oncotarget 7:86926-86936.

Wang Y, Chen Y, Liu S, Ye Y, Jiao H, Wang S, et al. 2016. MiR-384 inhibits human colorectal cancer metastasis by targeting KRAS and CDC42. Oncotarget 7:84826-84838.

Xia F, Sun J, Jiang Y, Li C. 2018. MicroRNA-384-3p inhibits retinal neovascularization through targeting hexokinase 2 in mice with diabetic retinopathy. Journal of cellular physiology 234:721-730.

Xia P, Liu Y, Cheng Z. 2016. Signaling Pathways in Cardiac Myocyte Apoptosis. BioMed research international 2016:9583268.

Xiao J, Shen B, Li J, Lv D, Zhao Y, Wang F, et al. 2014. Serum microRNA-499 and microRNA-208a as biomarkers of acute myocardial infarction. International journal of clinical and experimental medicine $7: 136$.

Yu N, Kakunda M, Pham V, Lill J, Du P, Wongchenko M, et al. 2015. HSP105 recruits protein phosphatase $2 \mathrm{~A}$ to dephosphorylate $\beta$-catenin. Molecular and cellular biology 35:1390-1400.

Zhang L, Chen X, Su T, Li H, Huang Q, Wu D, et al. 2015. Circulating miR-499 are novel and sensitive biomarker of acute myocardial infarction. Journal of thoracic disease 7:303-308.

Zhu Z, Zhu J, Zhao X, Yang K, Lu L, Zhang F, et al. 2015. All-trans retinoic acid ameliorates myocardial ischemia/reperfusion injury by reducing cardiomyocyte apoptosis. PLoS One 10:e133414. 